\title{
MODELING AND NUMERICAL SIMULATION OF SEMI-AUTOMATIC PISTOL DYNAMICS
}

\author{
Cyprian Suchocki, Janusz Ewertowski \\ Warsaw University of Technology, Department of Mechanics and Armament Technology, Warsaw, Poland \\ e-mail: c.suchocki@imik.wip.pw.edu.pl
}

In this study, a mathematical model of a semi-automatic hand-held weapon is presented. The dynamical equations of motion are derived by utilizing the formalism of Lagrange equations of the second kind. The model is applied to describe motion of P-64 semi-automatic pistol. The characteristics of rheological elements accounting for the mechanical reaction of the human hand are identified and are further used to determine the force and torque which act on the human hand during a shot. Some exemplary results of the conducted parametric study are presented in order to illustrate sensitivity of the system.

Keywords: pistol, firearm, recoil, pitch, muzzle climb

\section{Notations}

$a, b \quad$ - distance between slide and receiver mass centers perpendicular and parallel to barrel axis, respectively

$\mathbf{A}_{4 \times 4}, \mathbf{B}_{4 \times 4}, \mathbf{C}_{4 \times 4}-$ mass, damping and stiffness matrix

$\mathbf{b}_{4 \times 1}, \mathbf{F}_{4 \times 1}$ - force and external forces column matrix

$b_{c_{1}}, b_{c_{2}} \quad-$ viscosity coefficient of elasto-plastic and plastic collision

$b_{h x}, b_{h y}, b_{h \varphi}-$ hand damping coefficients

$c \quad-$ distance between slide mass center and barrel axis

$D \quad$ - Rayleigh dissipation function

$e_{1}, e_{2} \quad-$ length of undeformed Kelvin-Voigt element no. 1 and of undeformed damper no. 2

$F_{h}, M_{h} \quad-$ horizontal force and torque acting on human hand

$g \quad-$ gravitational acceleration

$H \quad-$ Heavisde step function

$J_{r}, J_{s} \quad-$ receiver and slide moment of inertia

$k, k_{c_{1}} \quad-$ recoil spring stiffness and stiffness coefficient of elasto-plastic collision

$k_{h x}, k_{h y}, k_{h \varphi}-$ hand stiffness coefficients

$m_{r}, m_{s} \quad-$ receiver and slide mass

$P_{s} \quad-$ breech force

$\mathbf{q} \quad-$ generalized coordinates column matrix, $\mathbf{q}=\left[q_{1}, q_{2}, q_{3}, q_{4}\right]^{\mathrm{T}}$

$\delta q_{j} \quad-j$-th generalized coordinate variation $(j=1,2,3,4)$

$Q, Q_{j}^{N} \quad$ - barrel pulling force and $j$-th non-potential generalized force $(j=1,2,3,4)$

$\mathbf{r}_{s}, \mathbf{r}_{r} \quad$ - slide and receiver mass center position vector

$\mathbf{r}_{r-s} \quad-$ position vector of slide mass center with respect to receiver mass center

$T, V \quad-$ total kinetic and total potential energy

$\mathbf{T}_{i-j} \quad-$ transformation matrix from $i$-th to $j$-th coordinate system $(i, j=0,1,2)$

$\mathbf{v}_{s}, \mathbf{v}_{r} \quad-$ slide and receiver mass center velocity vector

$\mathbf{v}_{r_{s}} \quad-$ slide velocity relative to receiver

$x_{r}, y_{r} \quad-$ horizontal and vertical coordinate of receiver mass center in inertial frame 
$x_{s} \quad-$ horizontal coordinate of slide mass center in inertial frame

$s, s_{\max }$ - slide translation and maximal slide translation

$\boldsymbol{\omega} \quad-$ pistol angular velocity

$\varphi \quad-$ angle of pistol rotation

$\delta A \quad-$ virtual work variation

\section{Introduction}

The recoil is a term used for the phenomenon of weapon motion which is caused by a gunshot (Fig. 1). Basically, two kinds of recoil can be distinguished (e.g. Hall, 2008):

- The horizontal recoil which refers to the weapon translation in the direction opposite to projectile motion.

- The vertical recoil, also called the pitch or the muzzle climb, which refers to the weapons rotation.

One may also adopt a different rule of distinction (e.g. Kochański 1979):

- The free recoil which takes place when shots are fired without physical contact of the firearm and the shooter (for instance, when the firearm is hung up on strings or rests on a surface which may roll on the floor with negligible friction).

- The braked recoil which takes place when the firearm is in contact with a part of the human body (e.g. hand or arm) during a shot. It results in the appearance of the so-called punch, i.e. a force is exerted by the weapon on the human organism during a gunshot and shortly after.

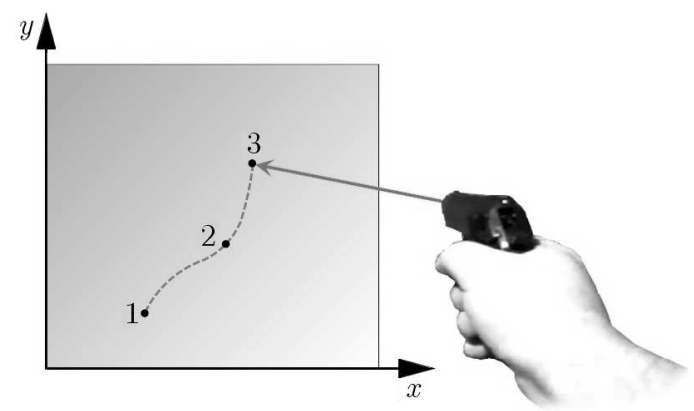

Fig. 1. Recoil of semi-automatic pistol

For semi-automatic weapons, one can also distinguish between the recoil and counterrecoil (e.g. Benzkofer, 1990). The term counterrecoil refers to the motion of weapon components when they return to their initial position, taken prior to the time instant when the shot took place.

The horizontal recoil is caused by the force which the burning propellant exerts on the gun. This force is commonly called the breech force. On the other hand, the reason for the vertical recoil is the existance of a certain distance between the barrel axis and the point at which the gun is supported. The distance between the breech force and the reaction force of the support results in the appearance of a torque which rotates the gun.

It is understandable that the human body has a limited mechanical strength and can bear punches only up to a certain limiting magnitude. Thus, if the firearm is incorrectly designed, the punch may cause bruises or even injuries.

The motion performed by a weapon during a shot may be of interest itself even without any investigation of the forces and torques acting on the hand holding the gun. During a gunshot, the pistol components are exposed to large accelerations mainly caused by the breech force and 
impacts. An inadequatly designed pistol may be damaged under such dynamic loadings, thus inflicting serious injuries on the person that is using it. A detailed motion and strength analysis of the firearm becomes an issue of great importance as far as safety nad proper operation are concerned. A numerical multibody simulation of semi-automatic pistol motion provides valuable input data for further local finite element analysis of a particular pistol part.

The literature concerning the recoil in semi-automatic pistols is relatively modest. A two-dimensional model of P-64 semi-automatic pistol was proposed by Czepukajtis (1974). The Lagrange equations method was utilized to derive the dynamical equations of motion. The post-impact velocities of the colliding pistol parts were calculated using the conservation of linear and angular momentum equations. A solution to the equations of motion was obtained only for a simplified version of the model. Some major discrepancies were observed between the simulation results and the experimental data.

Benzkofer (1990) investigated both horizontal and vertical recoil for M9 semi-automatic pistol. For that purpose, a two-dimensional multibody model was developed in DADS multibody dynamics software. The weapon components were modeled as rigid bodies with the incorporation of several springs and dampers to account for elasto-plastic and ideally elastic impacts. The pistol-human hand interaction was modeled by utilizing a single Kelvin-Voigt rheological element. The pistol motion following a single shot was simulated. A number of discrepancies between the experimental data and the simulation results were observed. In particular, the predictions of slide and receiver displacements were unsatisfactory. The author concluded that inadequate stiffness value assumed for the recoil spring is probably the major reason. What is more, the values of stiffness and damping coefficients assumed for the pistol-hand contact were considered to be too high, which resulted in very small receiver displacements.

A biomechanical model of a pistol grip handtool and human hand was developed by Lin et al. (2001). For that purpose, the tool-hand contact was modeled by utilizing several rheological elements. The model was mainly intended to simulate the motion of small hand tools such as the drill driver, for instance.

In this paper, a discrete model of a semi-automatic pistol is presented. The model takes into account the pistol-hand interaction which takes place during a shot. The equations of motion are derived utilizing the formalism of Lagrange equations of the second kind and represent a mechanical system with unilateral constraints. The equations of motion are transformed to the matrix form which is more suitable for performing numerical integration. The developed model is further applied to simulate the recoil of P-64 semi-automatic pistol. The experimental data gathered by Czepukajtis (1974) are used to calibrate the values of damping and stiffness coefficients. Finally, some exemplary parametric study results are presented that have been obtained for simulations of a series of subsequent shots.

\section{Semi-automatic pistol definition and operation}

A firearm is called semi-automatic if it uses a part of the recoil energy to extract the shell case, eject it and place a new cartridge into the chamber (Kochański, 1989). The principle of operation of a semi-automatic pistol will be explained utilizing the example of P-64 semi-automatic pistol (Fig. 2).

The most important components of the pistol are depicted in Fig. 3. One can distinguish the following phases of the pistol operation (Czepukajtis, 1974):

1. The trigger is pulled by the shooter which causes the firing pin to fire the primer. The propellant gases begin to expand which makes the projectile start moving down the barrel. Simultaneously, the gases exert the so-called breech force on the bottom surface of the 


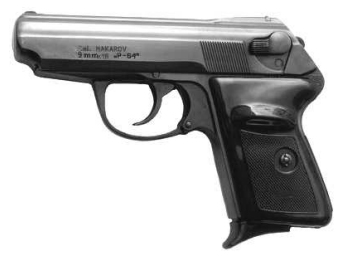

Fig. 2. P-64 semi-automatic pistol

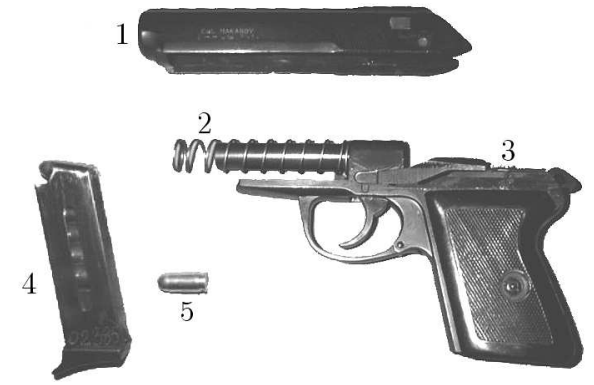

Fig. 3. P-64 pistol componentry: 1 - slide, 2 - recoil spring, 3 - receiver, 4 - magazine, 5 - projectile

cartidge case causing the backward movement of the slide. During its recoil, the slide compresses the recoil spring.

2. As the projectile opens the barrel, the breech force practically drops to zero. At the same time the slide continues to move backward. The spent cartridge is extracted and ejected.

3. The slides collides the receiver.

4. The impact, together with the energy accumulated in the compressed recoil spring, cause the slide to start moving to its forward position.

5. The slide continues its counterrecoil and picks up a new cartridge from the magazine.

6. The slide along with the new cartridge collide the receiver. The cartridge is pushed into the chamber. The slide stops in the forward position.

The system of operations listed above is typical for the group of blowback operated pistols. Another common design are recoil operated pistols. The only difference between a blowback and a recoil operated pistol is that in the case of the latter the slide is initially locked and unlocks after the bullet has left the barrel. The locking of the slide ensures that it will not open the breech while the projectile is still in the barrel. A premature opening of the breech would result in the propellant gases expanding in the shooter's hand and possibly disassembly the pistol.

\section{Physical model of a semi-automatic pistol and kinematics}

For the purpose of developing a physical model of a semi-automatic pistol, a number of assumptions is adopted, i.e.:

- The pistol displacement and rotation in the horizontal plane are assumed to be negligible. The motion of the mechanical system is two-dimensional and takes place in the vertical plane which contains the barrel axis and all mass centers.

- The mechanical system includes the receiver, the slide and the recoil spring with negligible mass. The slide is in motion with respect to the receiver (Fig. 4).

- The receiver and the magazine are modeled as a single rigid body which is referred to as receiver further in the text. The mass and the moment of inertia correspond to the magazine loaded with three cartridges. 


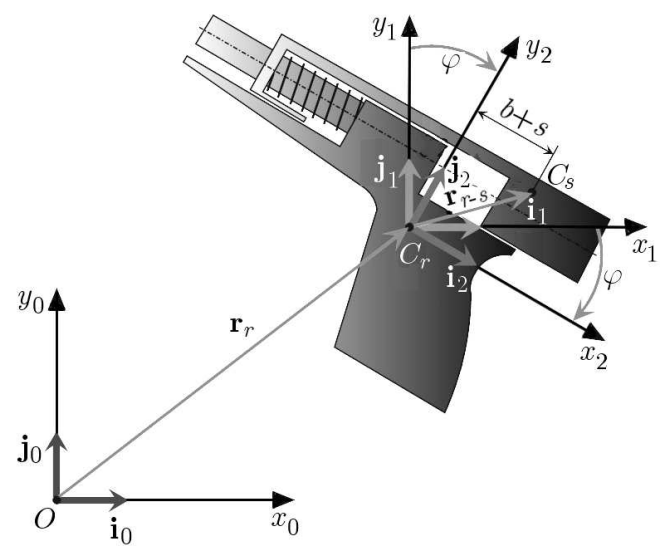

Fig. 4. Physical model and assumed coordinate systems

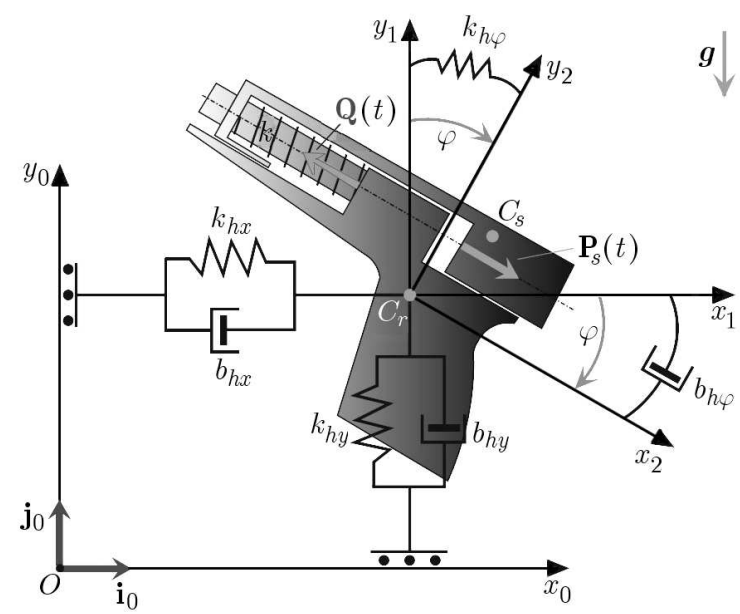

Fig. 5. Forces acting on the system and the model of contact
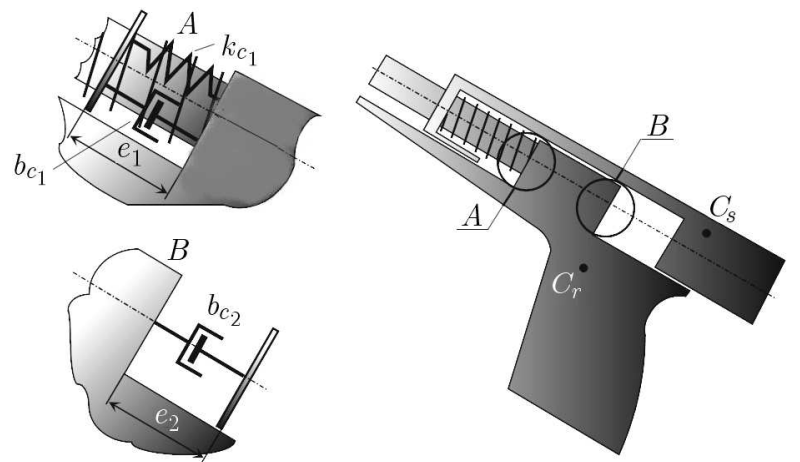

Fig. 6. Modeling of impact

- The human hand - pistol interaction is modeled by utilizing horizontal and vertical Kelvin-Voigt elements along with an angular spring and an angular damper (Fig. 5). The strains exhibited by soft tissues of the human hand are assumed to be small, thus all the utilized springs and dampers are linear.

- The external forces acting on the pistol are the breech force $\mathbf{P}_{s}(t)$ and the force $\mathbf{Q}(t)$ which is exerted on the barrel by the projectile (Fig. 5). The force value estimations given in Czepukajtis (1974) are used.

- The slide-receiver impacts are simulated using very stiff rheological elements (Fig. 6), cf Kukla et al. (1992). 
- After the slide collides the receiver during its counterrecoil, they continue motion as a single rigid body.

To determine the motion of the pistol, the following orthogonal coordinate systems are introduced (Fig. 4):

$O x_{0} y_{0}$ - the inertial coordinate system,

$C_{r} x_{1} y_{1}$ - the system parallel to the inertial coordinate system with the origin fixed at the mass center of the receiver,

$C_{r} x_{2} y_{2}$ - the system fixed to the receiver with the origin at the receiver mass center.

The basis vectors of the systems $C_{r} x_{1} y_{1}$ and $O x_{0} y_{0}$ are related as in

$$
\left\{\begin{array}{l}
\mathbf{i}_{1} \\
\mathbf{j}_{1}
\end{array}\right\}=\mathbf{T}_{0-1}\left\{\begin{array}{l}
\mathbf{i}_{0} \\
\mathbf{j}_{0}
\end{array}\right\} \quad \mathbf{T}_{0-1}=\left[\begin{array}{ll}
1 & 0 \\
0 & 1
\end{array}\right]
$$

whereas for the basis vectors of the systems $C_{r} x_{2} y_{2}$ and $C_{r} x_{1} y_{1}$

$$
\left\{\begin{array}{c}
\mathbf{i}_{2} \\
\mathbf{j}_{2}
\end{array}\right\}=\mathbf{T}_{1-2}\left\{\begin{array}{c}
\mathbf{i}_{1} \\
\mathbf{j}_{1}
\end{array}\right\} \quad\left\{\begin{array}{l}
\mathbf{i}_{1} \\
\mathbf{j}_{1}
\end{array}\right\}=\mathbf{T}_{2-1}\left\{\begin{array}{l}
\mathbf{i}_{2} \\
\mathbf{j}_{2}
\end{array}\right\}
$$

with

$$
\mathbf{T}_{1-2}=\left[\begin{array}{cc}
\cos \varphi & -\sin \varphi \\
\sin \varphi & \cos \varphi
\end{array}\right] \quad \mathbf{T}_{2-1}=\mathbf{T}_{1-2}^{\mathrm{T}}
$$

and

$$
\mathbf{T}_{0-2}=\mathbf{T}_{1-2} \mathbf{T}_{0-1} \quad \mathbf{T}_{2-0}=\mathbf{T}_{0-2}^{\mathrm{T}}=\mathbf{T}_{0-1}^{\mathrm{T}} \mathbf{T}_{1-2}^{\mathrm{T}}
$$

The position vector of the receiver mass center $C_{r}$ (Fig. 4) is

$$
\mathbf{r}_{r}=x_{r} \mathbf{i}_{1}+y_{r} \mathbf{j}_{1}
$$

whereas the position vector of the slide mass center $C_{s}$ is

$$
\mathbf{r}_{s}=\mathbf{r}_{r}+\mathbf{r}_{r-s} \quad \mathbf{r}_{r-s}=(b+s) \mathbf{i}_{2}+a \mathbf{j}_{2}
$$

The velocity of $C_{r}$ is given by the relation

$$
\mathbf{v}_{r}=\dot{x}_{r} \mathbf{i}_{1}+\dot{y}_{r} \mathbf{j}_{1}
$$

while the velocity of $C_{s}$

$$
\mathbf{v}_{s}=\mathbf{v}_{r}+\boldsymbol{\omega} \times \mathbf{r}_{r-s}+\mathbf{v}_{r_{s}} \quad \boldsymbol{\omega}=-\dot{\varphi} \mathbf{k}_{2} \quad \mathbf{v}_{r_{s}}=\ddot{s} \mathbf{i}_{2}
$$

After calculating the cross product in $(3.8)_{1}$, the following equation is found

$$
\mathbf{v}_{s}=\left(\dot{x}_{r} \cos \varphi-\dot{y}_{r} \sin \varphi+\dot{\varphi} a+\dot{s}\right) \dot{\mathbf{i}}_{2}+\left(\dot{x}_{r} \sin \varphi+\dot{y}_{r} \cos \varphi-\dot{\varphi}(b+s)\right) \mathbf{j}_{2}
$$

The mechanical system has four degrees of freedom, i.e. $x_{r}, y_{r}, \varphi$ and $s$. 


\section{Dynamical model of the semi-automatic pistol}

The Lagrange equations of the second kind in the presence of potential and dissipative forces take the form (e.g. Gutowski, 1971)

$$
\frac{\mathrm{d}}{\mathrm{d} t}\left(\frac{\partial T}{\partial \dot{q}_{j}}\right)-\frac{\partial T}{\partial q_{j}}=\frac{\partial V}{\partial q_{j}}-\frac{\partial D}{\partial \dot{q}_{j}}+Q_{j}^{N} \quad j=1,2, \ldots, 4
$$

where

$$
q_{1}=\varphi \quad q_{2}=x_{r} \quad q_{3}=y_{r} \quad q_{4}=s
$$

According to König's theorem, the total kinetic energy of the system is

$$
T=\frac{1}{2} m_{r} \mathbf{v}_{r} \cdot \mathbf{v}_{r}+\frac{1}{2} m_{s} \mathbf{v}_{s} \cdot \mathbf{v}_{s}+\frac{1}{2}\left(J_{r}+J_{s}\right) \dot{\varphi}^{2}
$$

After substituting (3.7) and (3.9) into (4.2), the energy takes form

$$
\begin{aligned}
T= & \frac{1}{2} m_{r}\left(\dot{x}_{r}^{2}+\dot{y}_{r}^{2}\right)+\frac{1}{2}\left(J_{r}+J_{s}\right) \dot{\varphi}^{2} \\
& +\frac{1}{2} m_{s}\left[\left(\dot{x}_{r} \cos \varphi-\dot{y}_{r} \sin \varphi+\dot{\varphi} a+\dot{s}\right)^{2}+\left(\dot{x}_{r} \sin \varphi+\dot{y}_{r} \cos \varphi-\dot{\varphi}(b+s)\right)^{2}\right]
\end{aligned}
$$

By substitution of (4.3) into (4.1) and performing differentiations, one obtains

$$
\begin{aligned}
& {\left[J_{r}+J_{s}+m_{s}\left((b+s)^{2}+a^{2}\right)\right] \ddot{\varphi}+m_{s}[a \cos \varphi-(b+s) \sin \varphi] \ddot{x}_{r}} \\
& \quad-m_{s}[a \sin \varphi+(b+s) \cos \varphi] \ddot{y}_{r}+m_{s} a \ddot{s}+2 m_{s}(s+b) \dot{\varphi} \dot{s}=Q_{\varphi} \\
& m_{s}[a \cos \varphi-(b+s) \sin \varphi] \ddot{\varphi}+\left(m_{r}+m_{s}\right) \ddot{x}_{r}+m_{s} \cos \varphi \ddot{s} \\
& \quad-m_{s}[a \sin \varphi+(b+s) \cos \varphi] \dot{\varphi}^{2}-2 m_{s} \dot{\varphi} \dot{s} \sin \varphi=Q_{x_{r}} \\
& -m_{s}[a \sin \varphi+(b+s) \cos \varphi] \ddot{\varphi}+\left(m_{r}+m_{s}\right) \ddot{y}_{r}-m_{s} \sin \varphi \ddot{s} \\
& \quad+m_{s}[(b+s) \sin \varphi-a \cos \varphi] \dot{\varphi}^{2}-2 m_{s} \dot{\varphi} \dot{s} \cos \varphi=Q_{y_{r}} \\
& m_{s} a \ddot{\varphi}+m_{s} \cos \varphi \ddot{x}_{r}-m_{s} \sin \varphi \ddot{y}_{r}+m_{s} \ddot{s}-m_{s}(s+b) \dot{\varphi}^{2}=Q_{s}
\end{aligned}
$$

The potential energy of the system is

$$
V=m_{r} g y_{r}+m_{s} g\left[y_{r}-(b+s) \sin \varphi+a \cos \varphi\right]+\frac{1}{2} k s^{2}
$$

and the Rayleigh dissipation function is

$$
D=\frac{1}{2} b_{h \varphi} \dot{\varphi}^{2}+\frac{1}{2} b_{h x} \dot{x}_{r}^{2}+\frac{1}{2} b_{h y} \dot{y}_{r}^{2}
$$

The non-potential generalized forces, not included in the dissipation function, are determined using the principle of virtual work, i.e.

$$
\delta A=\sum_{j=1}^{4} Q_{j}^{N} \delta q_{j}
$$

which yields

$$
\begin{aligned}
& Q_{\varphi}^{N}=(a-c)\left[P_{s}(t)-Q(t)\right] \quad Q_{x_{r}}^{N}=\left[P_{s}(t)-Q(t)\right] \cos \varphi \\
& Q_{y_{r}}^{N}=-\left[P_{s}(t)-Q(t)\right] \sin \varphi \\
& Q_{s}^{N}=\left[-k_{c_{1}}\left(s-\left(s_{\max }-e_{1}\right)\right)-b_{c_{1}} \dot{s}\right] H\left(s-\left(s_{\max }-e_{1}\right)\right)-b_{c_{2}} \dot{s} H\left(e_{2}-s\right)+P_{s}(t)
\end{aligned}
$$

The impact forces arising from unilateral constrains are incorporated by utilization of certain Heaviside functions (Kukla et al., 1992). After the collision during the counterrecoil, the system continues its motion as a single rigid body with three degrees of freedom, i.e. $x_{r}, y_{r}$ and $\varphi$. 
The computer algebra software wxMaxima has been used to write a script further utilized to generate the equations of motion of the system (Ney de Souza et al., 2004).

\section{Numerical integration}

For the purpose of numerical integration, the equations of motion are written in the matrix form (e.g. Grabysz, 2002), i.e.

$$
\mathbf{A}_{4 \times 4} \ddot{\mathbf{q}}_{4 \times 1}+\mathbf{B}_{4 \times 4} \dot{\mathbf{q}}_{4 \times 1}+\mathbf{C}_{4 \times 4} \mathbf{q}_{4 \times 1}=\mathbf{F}_{4 \times 1}
$$

Introducing a column matrix

$$
\mathbf{b}_{4 \times 1}=\mathbf{F}_{4 \times 1}-\mathbf{B}_{4 \times 4} \dot{\mathbf{q}}_{4 \times 1}-\mathbf{C}_{4 \times 4} \mathbf{q}_{4 \times 1}
$$

Thus

$$
\mathbf{A}_{4 \times 4} \ddot{\mathbf{q}}_{4 \times 1}=\mathbf{b}_{4 \times 1}
$$

The matrix components are found in wxMaxima symbolic algebra system by taking advantage of coeff command (Ney de Souza et al., 2004), i.e.

$$
\begin{aligned}
& A_{i j}=\operatorname{coeff}\left(\frac{\mathrm{d}}{\mathrm{d} t}\left(\frac{\partial T}{\partial \dot{q}_{i}}\right), \ddot{q}_{j}\right) \\
& b_{i}=Q_{i}^{N}-\frac{\partial V}{\partial q_{i}}-\frac{\partial D}{\partial \dot{q}_{i}}+\frac{\partial T}{\partial q_{i}}-\left[\frac{\mathrm{d}}{\mathrm{d} t}\left(\frac{\partial T}{\partial \dot{q}_{i}}\right)-\sum_{j=1}^{4} \operatorname{coeff}\left(\frac{\mathrm{d}}{\mathrm{d} t}\left(\frac{\partial T}{\partial \dot{q}_{i}}\right), \ddot{q}_{j}\right) \ddot{q}_{j}\right]
\end{aligned}
$$

Equation (5.3) takes an explicit form

$$
\left[\begin{array}{cccc}
A_{11} & A_{12} & A_{13} & A_{14} \\
A_{21} & A_{22} & 0 & A_{24} \\
A_{31} & 0 & A_{33} & A_{34} \\
A_{41} & A_{42} & A_{43} & A_{44}
\end{array}\right]\left\{\begin{array}{c}
\ddot{\varphi} \\
\ddot{x}_{r} \\
\ddot{y}_{r} \\
\ddot{s}
\end{array}\right\}=\left\{\begin{array}{l}
b_{1} \\
b_{2} \\
b_{3} \\
b_{4}
\end{array}\right\}
$$

with the following matrix components

$$
\begin{array}{llrl}
A_{11} & =J_{r}+J_{s}+m_{s}\left[(s+b)^{2}+a^{2}\right] \quad A_{12}=A_{21}=m_{s}[-(b+s) \sin \varphi+a \cos \varphi] \\
A_{13} & =A_{31}=-m_{s}[(b+s) \cos \varphi+a \sin \varphi] & A_{14}=A_{41}=m_{s} a \\
A_{22}=m_{r}+m_{s} & A_{23}=A_{32}=0 & A_{24}=A_{42}=m_{s} \cos \varphi \\
A_{33}=m_{r}+m_{s} & A_{34}=A_{43}=-m_{s} \sin \varphi & A_{44}=m_{s}
\end{array}
$$

and

$$
\begin{aligned}
b_{1}= & -2 m_{s}(b+s) \dot{\varphi} \dot{s}+m_{s} g[(b+s) \cos \varphi+a \sin \varphi]+(a-c)\left[P_{s}(t)-Q(t)\right]-k_{h \varphi} \varphi-b_{h \varphi} \dot{\varphi} \\
b_{2}= & 2 m_{s} \dot{\varphi} \dot{s} \sin \varphi+m_{s}[(b+s) \cos \varphi+a \sin \varphi] \dot{\varphi}^{2}+\left[P_{s}(t)-Q(t)\right] \cos \varphi-k_{h x} x_{r}-b_{h x} \dot{x}_{r} \\
b_{3}= & 2 m_{s} \dot{\varphi} \dot{s} \sin \varphi+m_{s}[-(b+s) \sin \varphi+a \cos \varphi] \dot{\varphi}^{2}-\left(m_{r}+m_{s}\right) g \\
& -\left[P_{s}(t)-Q(t)\right] \sin \varphi-k_{h y} y_{r}-b_{h y} \dot{y}_{r} \\
b_{4}= & {\left[-k_{c_{1}}\left(s-\left(s_{\max }-e_{1}\right)\right)-b_{c_{1}} \dot{s}\right] H\left(s-\left(s_{\max }-e_{1}\right)\right)-b_{c_{2}} \dot{s} H\left(e_{2}-s\right) } \\
& +m_{s}(b+s) \dot{\varphi}^{2}+m_{s} g \sin \varphi-k s+P_{s}(t)
\end{aligned}
$$

The given system of nonlinear ordinary differential equations has been solved in Scilab. For that purpose, a program has been written utilizing the Scilab programming language (e.g. Brozi, 2007). 


\section{Model calibration}

The developed mathematical model has been applied to simulate the motion of P-64 semi-automatic pistol. The experimental data gathered in Czepukajtis (1974) are utilized to determine values of the stiffness and damping coefficients responsible for slide-receiver collisions and pistol-hand contact. The results of the curve-fitting can be seen in Fig. 7. The time instant at which the bullet leaves the barrel has been marked with a vertical solid line.
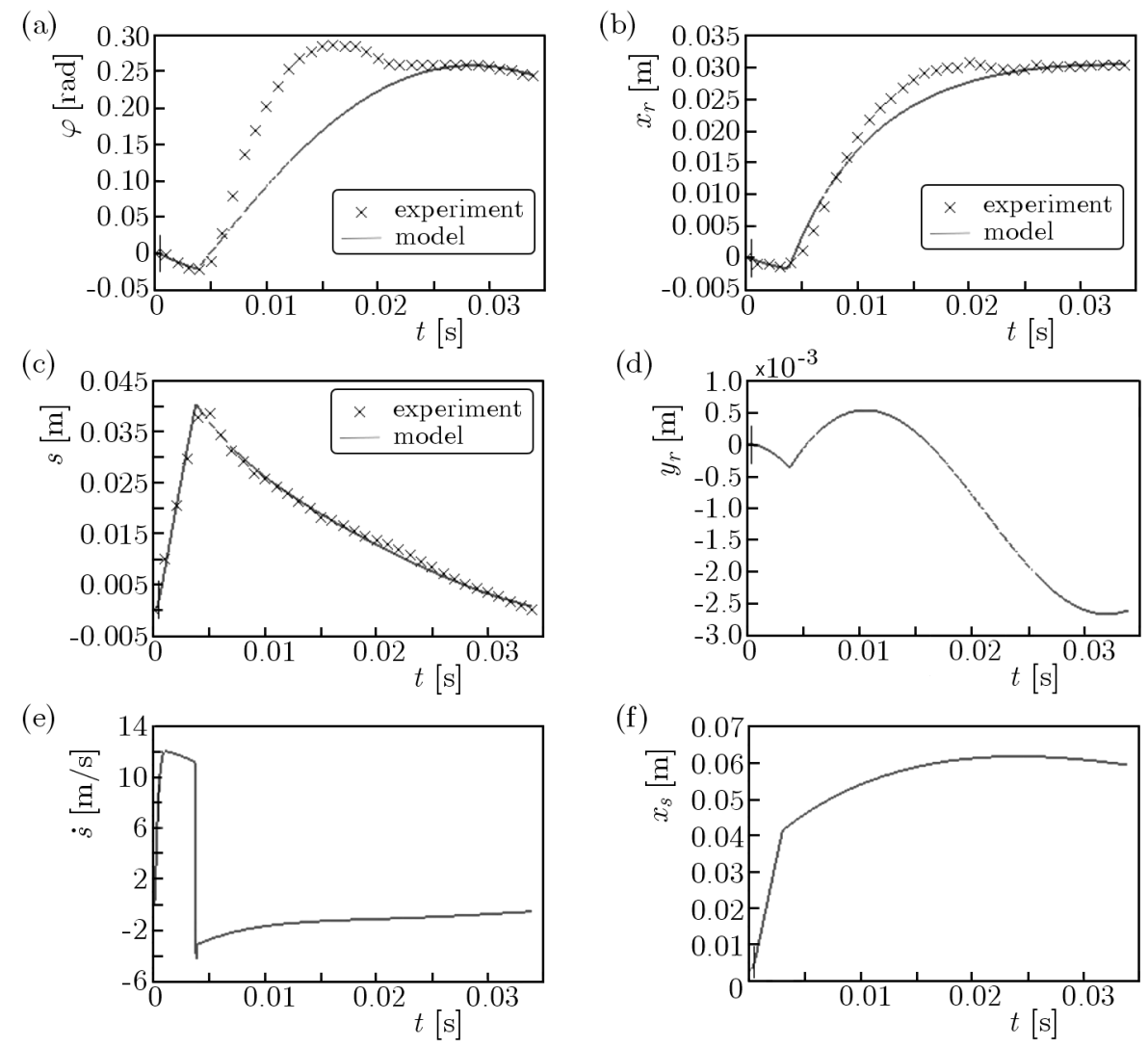

Fig. 7. Model predictions and experimental data
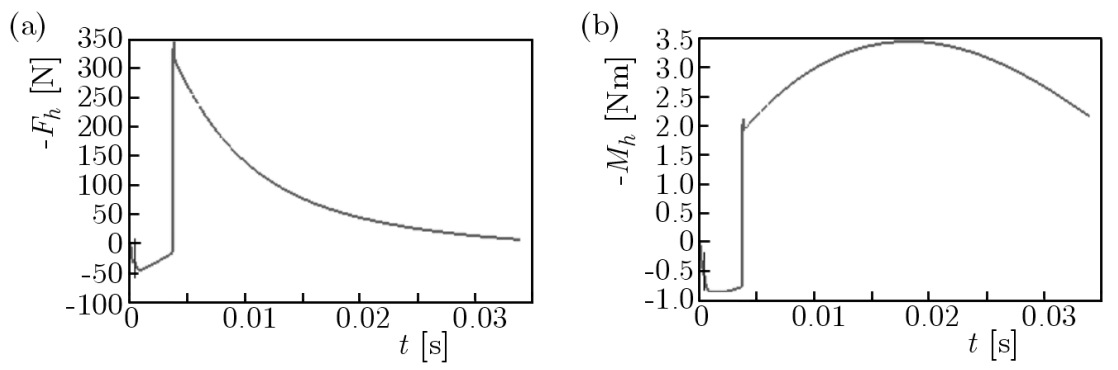

Fig. 8. Force and torque exerted on hand

\section{Exemplary results of parametric study}

The sensitivity has been investigated by performing simulations of a 4 -shot series. The subsequent shots have been assumed to occur after $\Delta t=0.25 \mathrm{~s}$. In Fig. 9. the simulation results obtained for various magnitudes of the breech force $P_{s}$ are presented. In Fig. 10, the simulation results obtained for different values of the recoil spring stiffness $k$ are presented. 
(a)

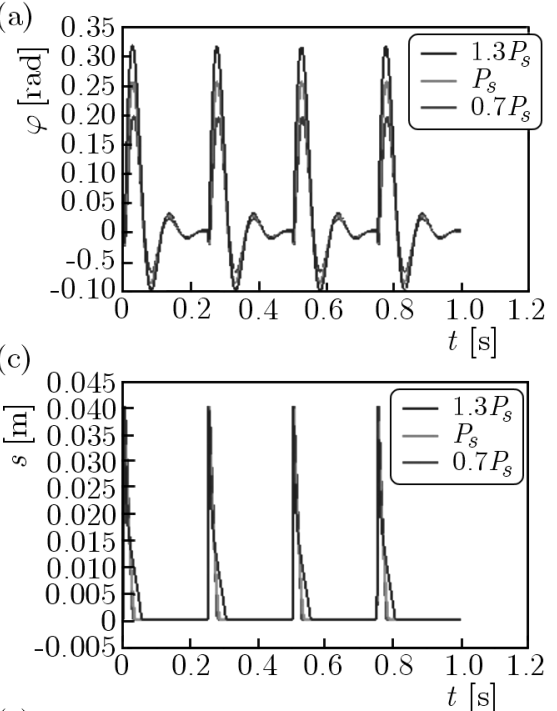

(e)

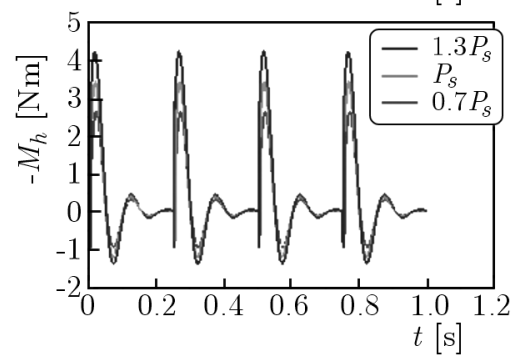

(b)

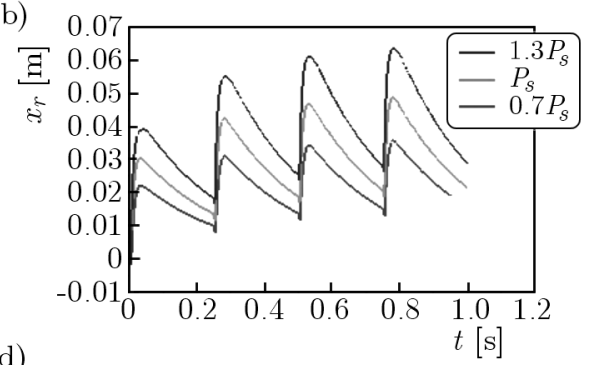

(d)

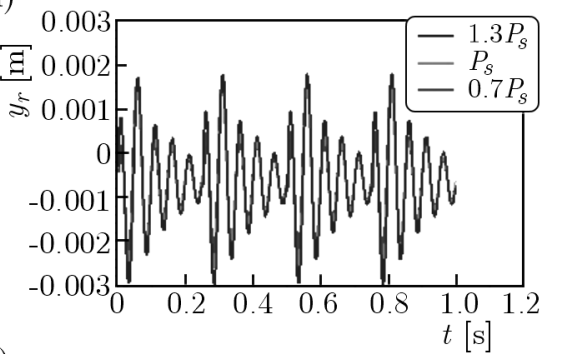

(f)

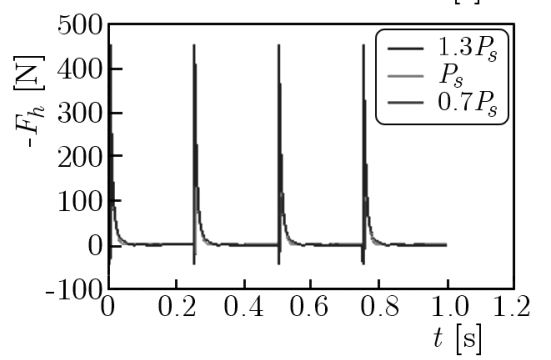

Fig. 9. Simulation results for four subsequent shots with varying magnitude of the breech force

(a)

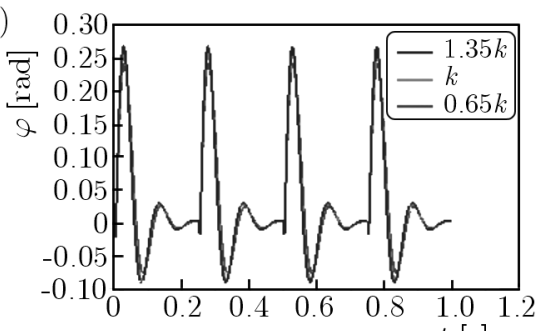

(c)

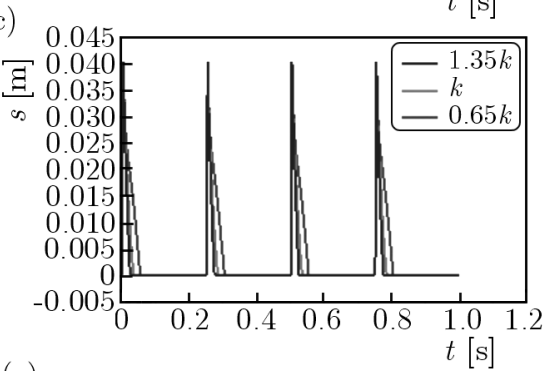

(e)

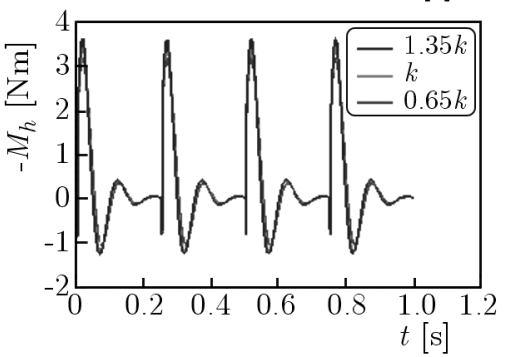

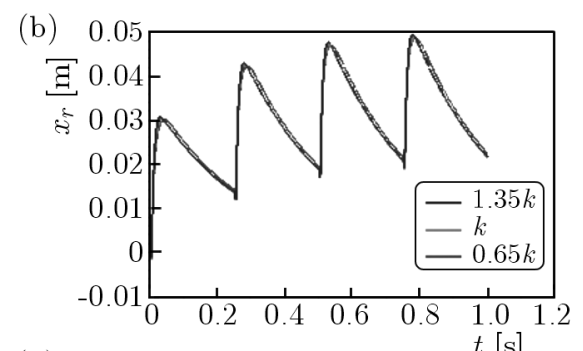

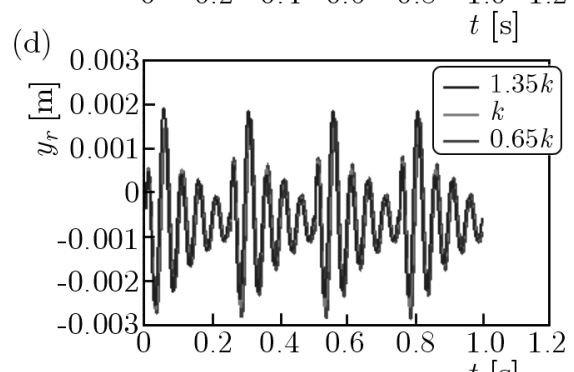

(f)

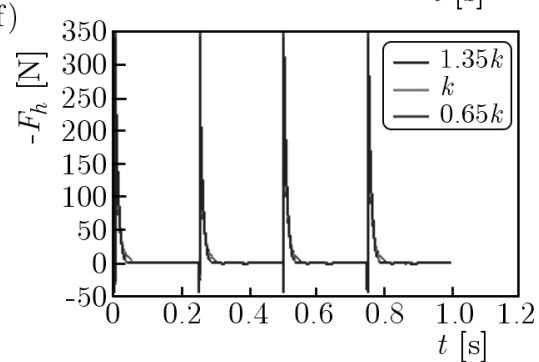

Fig. 10. Simulation results for four subsequent shots with varying stiffness of the recoil spring 


\section{Conclusions}

The results presented above allow one to make the following observations:

- The projectile leaves the barrel after approximately $0.375 \mathrm{~ms}$. At this time instant the pistol displacements caused by both horizontal and vertical recoil are negligible (Fig. 7). Thus, as far as a single shot is concerned, the recoil has no serious influence on shooting accuracy.

- A break of about $0.25 \mathrm{~s}$ is sufficient to almost completely damp out the pistols angular motion (Fig. 9a and Fig. 10a).

- The longitudinal displacements of the pistol in the case of several subsequent shots do accumulate (Fig. 9b and Fig. 10b). There is not enough time for the human hand to damp the longitudinal motion out.

- The magnitude of the breech force has a very strong influence on pistol displacements and the pistol-hand interaction (Fig. 9).

- The simulation results are in a good agreement with the experimental observation that the receiver displacements are much larger than the total displacements of the slide (Czepukajtis, 1974). In fact it is the receiver that hits an almost motionless slide during the last phase of the operation cycle, see Figs. $7 \mathrm{~b}$ and $7 \mathrm{f}$.

The aforementioned conclusions represent some exemplary information that the mathematical model of a semi-automatic pistol proposed in this study may provide a weapon designer with. The developed model is general and may be useful for simulating the recoil of both blowback operated and recoil operated handguns.

\section{References}

1. Benzkofer P.D., 1990, Dynamic analysis of a hand-held weapon, Proceedings of the Sixth U.S. Army Symposium on Gun Dynamics, 2, 62-79

2. Brozi A., 2007, Scilab in Examples (in Polish), Nakom Publishing, Poznań

3. Czepukajtis W., 1974, Influence of operator on motion of light mechanical devices (in Polish), $\mathrm{PhD}$ thesis, Institute of Mechanical Equipment Design, Warsaw University of Technology, Warsaw

4. Grabysz W., 2002, Selected Topics in Symbolic-Numerical Simulation of Mechanisms (in Polish), Silesian University of Technology Publishing, Gliwice

5. Gutowski R., 1971, Analytical Mechanics (in Polish), Polish Scientific Publishers, Warsaw

6. Hall M.J., 2008, Measuring felt recoil for sporting arms, International Journal of Impact Engineering, 35, 540-548

7. Kochański S., 1979, Braked recoil of firearms (in Polish), Scientific Surveys of the Institute of Mechanical Equipment Design, 2, Warsaw University of Technology, Warsaw

8. Kochański S., 1989, Low Caliber Automatic Weapons (in Polish), Warsaw University of Technology Publishing House, Warsaw

9. Kukla S., Posiadala B., Skalmierski B., Tylikowski M., 1992, Dynamics problem of mechanical systems with unilateral constraints, Journal of Theoretical and Applied Mechanics, 30, $401-418$

10. Lin J.-H., Radwin R.G., Richard T.G., 2001, Dynamic biomechanical model of the hand and arm in pistol grip power handtool usage, Ergonomics, 44, 295-312

11. Ney de Souza P., Fateman R.J., Moses J., Yapp C., 2004, The Maxima Book, on laws of the manuscript 